\title{
Relação mãe-filho e fatores associados: análise hierarquizada de base populacional em uma capital do Brasil-Estudo BRISA
}

\author{
Mother-child relationship and associated factors: Hierarchical \\ analysis of the population base in a Brazilian state capital \\ - BRISA Study
}

\author{
Milady Cutrim Vieira Cavalcante ${ }^{1}$ \\ Fernando Lamy Filho ${ }^{1}$ \\ Ana Karina Teixeira da Cunha França ${ }^{1}$ \\ Zeni Carvalho Lamy ${ }^{1}$
}

${ }^{1}$ Hospital Universitário, Universidade Federal do Maranhão. R. Barão de Itapary 227, Centro. 65020-070 São Luís MA Brasil.miladycutrim@ yahoo.com.br

\begin{abstract}
Several factors can interfere in the mother-child relationship. Studies about different maternal characteristics and this relationship are scarce; they mainly evaluate women with psychopathology and use simultaneous regression models with adjustment for multiple confounders. This study aimed to assess factors associated with losses in the mother-child relationship through a cohort of 3,215 mothers of children between 15 and 36 months of age. Losses in the mother-child relationship, assessed by the Postpartum Bonding Questionnaire, was the outcome variable and the explanatory variables were demographic, socioeconomic, reproductive health and mental health of mothers as well as the conditions of the birth of children. It used multivariate regression analysis with a hierarchical approach in which the hierarchical blocks were structured according to the influence on the mother-child relationship. The prevalence of losses in the mother-child relationship was high (12.6\%) and associated risk factors to lower maternal education $(R R=1.64)$, having unplanned pregnancy $(R R=1.42)$, consumption of alcoholic beverages during pregnancy $(R R=1.42)$ and maternal stress symptoms $(R R=$ $1.88)$ and depression $(R R=2.00)$. Education and elements related to mental health were risks for damage in the mother-child relationship.

Key words Mother-child relationship, Psychosocial impact, Association
\end{abstract}

Resumo Diversos fatores podem interferir na relação mãe-filho. Estudos sobre as diferentes características maternas e esta relação são escassos, avaliam principalmente mulheres com psicopatologias e utilizam modelos de regressão simultânea com ajuste para múltiplos confundidores. Este estudo objetivou avaliar fatores associados a prejuizos na relação mãe-filho por meio de uma coorte com 3.215 mães de crianças entre 15 e 36 meses de idade. Prejuizos na relação mãe-filho, avaliado pelo Postpartum Bonding Questionnaire, constituiu a variável desfecho e as variáveis explanatórias foram características demográficas, socioeconômicas, da saúde reprodutiva e da saúde mental das mães bem como das condições do nascimento das crianças. Foi utilizada análise de regressão multivariada com abordagem hierarquizada em que os blocos hierárquicos foram estruturados segundo influência na relação mãe-filho. A prevalência de prejuizos na relação mãe-filho foi elevada $(12,6 \%)$ e associada com fatores de risco à menor escolaridade da mãe $(R R=1,64)$, não ter planejado a gravidez $(R R=1,42)$, consumo de bebida alcoólica durante a gravidez $(R R=1,42)$ e sintomas maternos de estresse $(R R=1,88)$ e depressão $(R R=2,00)$. Escolaridade e elementos relacionados à saúde mental materna constituíram risco para prejuizos na relação mãe-filho.

Palavras-chave Relações mãe-filho, Impacto psicossocial, Associação 


\section{Introdução}

O desenvolvimento da relação mãe-bebê é de grande importância ${ }^{1}$, especialmente ao longo dos primeiros anos de vida, em que a criança necessita de um relacionamento afetivo contínuo para se desenvolver de modo saudável ${ }^{2}$. A existência de prejuízos nesta relação pode condicionar o desenvolvimento infantil ${ }^{3,4}$ gerando alterações nos níveis cognitivo, emocional e social ${ }^{2}$.

Prejuízos na relação mãe-filho dificilmente podem ser explicados mediante análise de um único fator isoladamente, pois as condições de risco tendem a ser multidimensionais ${ }^{5}$, envolvendo, com diferentes intensidades, aspectos socioculturais, biológicos e psicológicos referentes à mãe ou ao bebê.

A literatura tem retratado prejuízos na relação mãe-filho associados à menor renda e escolarida$\mathrm{de}^{7,8}$, pois, segundo Ferreira e Lima ${ }^{8}$ e Popp et al. ${ }^{9}$, mães com baixo nível socioeconômico podem apresentar maior incidência de padrões negativos quanto à capacidade de responder às necessidades da criança. Estes autores sugerem que, em contextos desfavoráveis, estas apresentariam maior dificuldade em reconhecer e responder aos sinais da criança, maior negatividade verbal, baixo calor afetivo, menor envolvimento e dariam menos suporte às atividades da criança. Ressaltam, ainda, que tais condições poderiam influenciar negativamente a qualidade da interação materna por constituírem fonte de estresse familiar ${ }^{8,9}$.

O tipo de parto também parece interferir na relação mãe-filho. Resultados robustos apontaram que mães submetidas à cesariana, sobretudo quando não planejada, expressaram reação inicial menos positiva com o bebê, que se manteve por até seis semanas ${ }^{10}$. A cesariana, que no Brasil se apresenta em proporção elevada, também colabora significativamente para a separação precoce mãe-bebê $\hat{~}^{11}$. Do mesmo modo, os partos instrumentais, por serem mais estressantes, associam-se a níveis mais elevados de cortisol nas mães ${ }^{12}$, podendo também comprometer a disponibilidade para a vinculação inicial ${ }^{13}$.

Outro aspecto que pode afetar negativamente a relação mãe-filho está relacionado a problemas de saúde mental materna ${ }^{14-16}$. Estudos têm demonstrado a influência negativa da depressão, da ansiedade e do estresse maternos sobre a saúde da mulher, a sua relação com a família e com o recém-nascido, dificultando o estabelecimento do vínculo mãe-bebê e gerando consequências para o desenvolvimento da criança sob vários aspectos $^{17,18}$.
Por outro lado, relações mais adequadas têm sido referidas em estudos cujos bebês foram amamentados ao seio ${ }^{19} \mathrm{e}$ cujos pais são casados, pois pais solteiros ou que somente coabitam tendem a ter relações mais instáveis afetando a capacidade das mães de proporcionar um ambiente estimulante, particularmente logo após o nascimento de uma criança, quando estão emocionalmente vulneráveis ${ }^{20}$. Segundo Klaus et al. ${ }^{19}$, a amamentação ao seio apresenta efeito tranquilizante sobre a mãe o que tende a aumentar seu elo com o bebê e deixá-la mais sensível.

A literatura tem constatado a influência de diversos fatores sobre a relação mãe-filho. Reck et al. ${ }^{21}$ avaliaram a associação entre prejuízos na relação mãe-filho, sintomas depressivos e características sociodemográficas em uma amostra populacional, na Alemanha. Sockol et al..$^{22}$ investigaram a correlação entre prejuízos na relação mãe-filho de 180 mulheres com depressão e características sociodemográficas, sintomas depressivos e fatores obstétricos destas. Entretanto, esses estudos são escassos, mais frequentes em mulheres com morbidades psicológicas ou psiquiátricas e utilizam abordagem estatística tradicional por meio de regressão simultânea com ajuste para múltiplos confundidores ${ }^{21,22}$.

Assim, a realização de estudo sobre a prevalência e elementos associados a prejuízos na relação mãe-filho, de base populacional e com utilização de modelos de análise hierarquizada pode ser mais apropriada para demonstrar as características desse fenômeno na população geral.

\section{Métodos}

Estudo de coorte que utilizou o banco de dados da pesquisa intitulada "Fatores etiológicos do nascimento pré-termo e consequências dos fatores perinatais na saúde da criança: Coortes de nascimentos em duas cidades brasileiras - BRISA".

O presente estudo incluiu 3.215 mães de crianças entre 15 e 36 meses de vida, residentes em São Luís, que possuíam dados completos quanto à avaliação da relação mãe-filho, realizada de abril de 2011 a março de 2013. Estas mães corresponderam ao seguimento da coorte de nascimento do BRISA, que entrevistou 5.167 mulheres imediatamente após o parto, no período de janeiro a dezembro de 2010, sendo a perda para o seguimento, portanto, de 1.952 crianças $(37,77 \%)$.

Para minimizar a influência dessa perda na análise, foi realizado procedimento de pondera- 
ção que levou em consideração as diferenças na distribuição de duas variáveis no grupo de perdas e no efetivamente analisado. A escolha dessas variáveis levou em consideração a ausência de colinearidade, além das diferentes características da população, sendo selecionada uma de caráter socioeconômico (classificação econômica) e outra, biológico (idade gestacional).

A amostra da coorte de nascimento (5.167) foi estratificada por maternidade, com quota proporcional ao número de partos anuais das mesmas, sendo excluídas aquelas com menos de 100 partos ao ano. O tamanho mínimo calculado da amostra do nascimento foi fixado em 5.000. Foi realizada amostragem sistemática, em que era sorteada uma mulher a cada três nascimentos.

Dados demográficos, socioeconômicos e de saúde reprodutiva maternos, foram obtidos na coorte de nascimento. Dados da saúde mental materna (sintomas de depressão e sintomas de estresse) e características referentes ao recémnascido e à relação mãe-filho foram obtidos no segmento do segundo ou terceiro anos de vida.

Para avaliação de sintomas de depressão entre as mães foi utilizada a Escala de Depressão Pós-Parto de Edimburgo (EPDS). Esta é a mais utilizada para rastreamento de sintomas depressivos que se manifestam após o parto por ser de fácil aplicação e interpretação. Foi proposta por Cox et al..$^{23}$ sendo posteriormente validada para a população brasileira por Santos et al. ${ }^{24}$. Consiste em um instrumento de autorresposta composta por dez itens, cujas opções são pontuadas de zero a três, de acordo com a presença ou intensidade do sintoma. O somatório dos pontos perfaz escore de 30 , sendo considerados de sintomatologia depressiva, valores iguais ou superiores a $12^{25}$.

Sintomas de estresse materno foram investigados por meio da Escala de Estresse Percebido (Perceived Stress Scale - PSS 14) proposta por Cohen e Williamson ${ }^{26}$ e validada para a população brasileira por Luft et al. ${ }^{27}$. Trata-se de uma medida global, autorreferida, que permite verificar em que grau as situações da vida de um indivíduo são percebidas como fatores estressores.

Esta escala é composta por 14 itens que relacionam acontecimentos e situações ocorridos nos últimos 30 dias com opções de respostas que variam de zero a quatro $(0=$ nunca; $1=$ quase nunca; 2 = às vezes; $3=$ quase sempre; $4=$ sempre). As questões com conotação positiva $(4,5,6$, $7,9,10$ e 13) têm sua pontuação somada invertida. As demais questões são negativas e devem ser somadas diretamente. A soma da pontuação das questões fornece escores que podem variar de zero (sem sintomas de estresse) a 56 (sintomas de estresse extremo $)^{27}$. Neste estudo, valores menores ou iguais a 30 representaram ausência de sintomas de estresse materno e maiores que este valor a presença de sintomas de estresse ${ }^{26}$.

"Prejuízos na relação mãe-filho" constituiu a variável desfecho, enquanto as variáveis explanatórias, obtidas a partir de um questionário estruturado, estão descritas no Quadro 1.

Para avaliação de prejuízos na relação mãe-filho, optou-se pelo Postpartum Bonding Questionnaire $(P B Q)$, útil para o diagnóstico de transtornos nesta relação ${ }^{3}$ e avaliado com bons níveis de sensibilidade ${ }^{28}$.

Utilizou-se a versão portuguesa do $P B Q^{29}$, instrumento de autorresposta cuja versão final possui 25 itens em uma escala de Likert: "sempre”, "muitas vezes", "frequentemente", “às vezes”, "raramente" e "nunca", variando de zero a cinco ${ }^{3}$, em que maiores valores são atribuídos às respostas consideradas indicativas de dificuldades na relação e menores valores às respostas que traduzem situações favoráveis a esta. Em alguns itens foi necessário inverter o sentido da valoração (itens 2, 3, 5, 6, 7, 10, 12, 13, 14, 15, 17, 18, 19, 20, $21,23$ e 24$)^{30}$.

A maioria dos estudos utiliza este instrumento a partir da formação de quatro fatores. No presente estudo utilizou-se apenas o primeiro fator da escala original ${ }^{3}$, por permitir comparações com outras investigações, visto que a opção de considerar apenas o primeiro fator de Brockington et al. ${ }^{3}$ é partilhada por outros autores ${ }^{26}$ por constituir o único fator original com valores de consistência interna aceitáveis nos diversos estudos realizados ${ }^{21,31}$ e por ser considerado o mais adequado para avaliar a população geral ${ }^{21,32}$.

Denominado "ligação ao bebê danificada", o primeiro fator se refere à ausência ou à fraca intensidade de sentimentos positivos e de proximidade com o bebê, sendo constituído pelo agrupamento de doze itens. Este apresenta variação de 0 a 60 , com pontos de corte $<12$ considerado normal e $\geq 12$, patológico $^{29}$.

$\mathrm{Na}$ análise descritiva, as variáveis categóricas foram apresentadas por meio de frequências e porcentagens. A confiabilidade do $P B Q$ revelou boa consistência interna verificada com coeficiente alfa de Cronbach 0,8.

Para identificação dos fatores de risco associados a prejuízos na relação mãe-filho foi utilizada análise de regressão multivariada com abordagem hierarquizada, cujo modelo teórico foi formado por quatro blocos de variáveis e encontra-se representado no Quadro 1. 


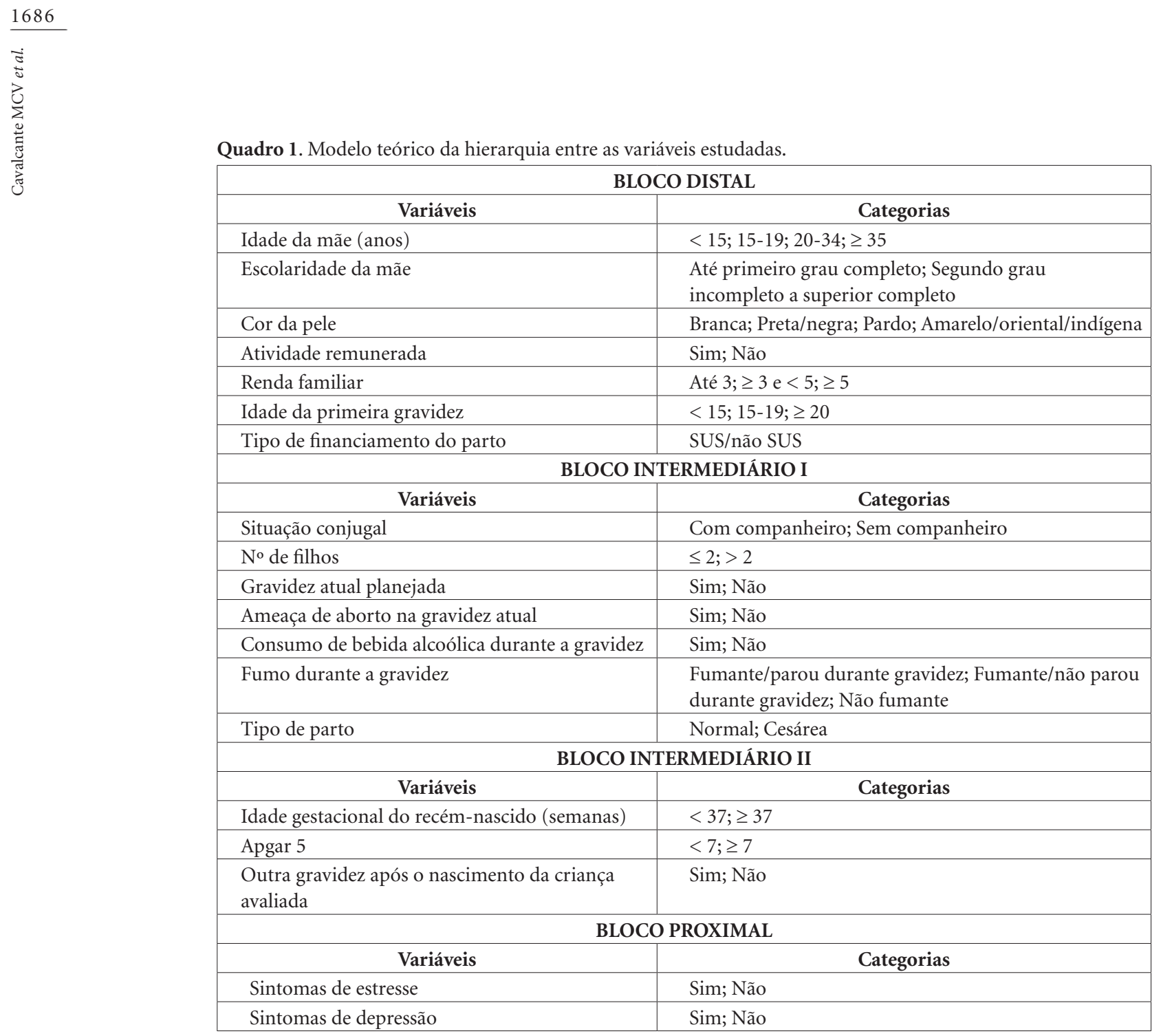

A escolha das variáveis de cada bloco não levou em consideração a classificação habitual das variáveis (demográficas, socioeconômicas, hábitos de vida etc.), mas seu suposto nível de influência em relação ao desfecho.

No primeiro bloco, o mais distal, foram agrupadas as variáveis idade da mãe, escolaridade, cor da pele, atividade remunerada, renda familiar, idade da primeira gestação e tipo de financiamento do parto. O segundo foi composto por variáveis demográficas, da saúde reprodutiva e hábitos de vida. Características relativas ao recém-nascido e à variável 'outra gravidez após o nascimento da criança avaliada' constituíram as variáveis do terceiro bloco. No quarto bloco, considerado proximal ao desfecho, foram incluídas apenas variáveis relativas à saúde mental materna
- estresse e depressão - por serem consideradas de elevada influência na qualidade da relação mãe-filho.

As variáveis que apresentaram valor de $\mathrm{p}<$ 0,05 em qualquer nível do modelo teórico foram consideradas significantes e passaram a compor o grupo das variáveis do nível seguinte. Este procedimento foi repetido até o nível final.

Também foram estimados o risco relativo (RR) e seus respectivos intervalos de confiança, de $95 \%$. Os dados foram analisados no programa estatístico Stata 12.0.

O BRISA obteve aprovação do Comitê de Ética em Pesquisa do Hospital Universitário da UFMA e do Hospital das Clínicas da Faculdade de Medicina de Ribeirão Preto. 


\section{Resultados}

A Tabela 1 apresenta as diferenças de características entre o grupo de perdas e o grupo que permaneceu no estudo.

A taxa de resposta do estudo foi de $62,2 \%$ $(\mathrm{n}=3.215)$ em relação às mães entrevistadas na ocasião do nascimento de seus filhos $(\mathrm{n}=5.167)$, sendo as perdas decorrentes, principalmente, de recusa e dificuldade de localização das mães.

Predominaram mães na faixa etária de 2034 anos $(73,7 \%)$, com companheiro $(80,6 \%)$, de cor da pele parda $(67,7 \%)$, que possuíam até dois filhos $(80,8 \%)$, com segundo grau incompleto a superior completo $(76,6 \%)$ e que recebiam até três salários mínimos $(66,6 \%)$. A porcentagem de fumo durante a gravidez foi de $6,7 \%$ e de consumo de bebida alcoólica de 14,0\% durante esse período (Tabela 2).
Características dos recém-nascidos e da vida reprodutiva das mulheres estudadas estão descritas na Tabela 3. Verificou-se que $43,1 \%$ das mães tiveram a primeira gravidez quando estavam entre 15 e 19 anos de idade. Durante a gravidez atual, 7,5\% das mães sofreram ameaça de aborto. O tipo de parto normal ocorreu em 50,7\% dos casos. Entre os recém-nascidos, $85,9 \%$ nasceram a termo e somente $1,2 \%$ apresentaram valor de Apgar no quinto minuto menor ou igual a seis.

Verificou-se que $17,8 \%$ das entrevistadas apresentaram sintomas de estresse e $7,5 \%$ de depressão. A prevalência de prejuízos na relação mãe-filho detectada pelo $P B Q$ foi de $12,6 \%$ (dado não apresentado em tabela).

A Tabela 4 apresenta os fatores que estiveram associados a prejuízos na relação mãe-filho nos diferentes blocos de análise. No bloco mais distal de determinação, primeiro nível, a variável signi-

Tabela 1. Diferenças de características entre o grupo de perdas e o grupo que permaneceu no estudo, São Luís, 2013.

\begin{tabular}{|c|c|c|c|}
\hline Variáveis & $\begin{array}{c}\text { Grupo estudado } \\
\text { n (\%) }\end{array}$ & $\begin{array}{c}\text { Grupo de perdas } \\
\text { n }(\%)\end{array}$ & p-valor \\
\hline Escolaridade da mãe (anos de estudo)* & & & $<0,001$ \\
\hline 1 a 4 & $102(43,6)$ & $132(56,4)$ & \\
\hline 5 a 8 & $637(55,1)$ & $519(44,9)$ & \\
\hline 9 a 11 & $2.015(67,5)$ & $969(32,5)$ & \\
\hline$\geq 12$ & $457(58,7)$ & $322(41,3)$ & \\
\hline Renda familiar (SM) & & & $<0,001$ \\
\hline$\leq 1$ & $432(56,3)$ & $336(43,7)$ & \\
\hline$>1 \mathrm{e} \leq 3$ & $1.369(65,9)$ & $709(34,1)$ & \\
\hline$>3$ & $872(62,5)$ & $524(37,5)$ & \\
\hline Não sabe & $542(58,6)$ & $383(41,4)$ & \\
\hline Classificação econômica (CCEB) & & & $<0,001$ \\
\hline A & $53(36,5)$ & $92(63,5)$ & \\
\hline $\mathrm{B}$ & $494(62,7)$ & $294(37,3)$ & \\
\hline $\mathrm{C}$ & $1.741(66,9)$ & $863(33,1)$ & \\
\hline $\mathrm{D}$ & $670(59,6)$ & $455(40,4)$ & \\
\hline $\mathrm{E}$ & $89(45,6)$ & $106(54,4)$ & \\
\hline Ignorado & $168(54,2)$ & $142(45,8)$ & \\
\hline Paridade & & & 0,001 \\
\hline 1 & $1.543(63,2)$ & $900(36,8)$ & \\
\hline 2 a 4 & $1.581(62,2)$ & $960(37,8)$ & \\
\hline$\geq 5$ & $91(49,7)$ & $92(50,3)$ & \\
\hline Idade Gestacional (semanas) & & & 0,001 \\
\hline$<37$ & $377(56,3)$ & $293(43,7)$ & \\
\hline$\geq 37$ & $2.838(63,1)$ & $1.659(36,9)$ & \\
\hline Peso ao nascer ${ }^{\star}$ (gramas) & & & 0,002 \\
\hline$<2.500$ & $248(55,6)$ & $198(44,4)$ & \\
\hline$\geq 2.500$ & $2.960(62,9)$ & $1.745(37,1)$ & \\
\hline
\end{tabular}

${ }^{*} \mathrm{n}$ inferior a 3.215 no grupo estudado. SM - Salários Mínimos. CCEB - Critério de Classificação Econômica Brasil. 
Tabela 2. Características demográficas, socioeconômicas e de hábitos de vida das mulheres estudadas, São Luís, 2013.

\begin{tabular}{|c|c|}
\hline Variáveis & n (\%) \\
\hline \multicolumn{2}{|l|}{ Idade da mãe (anos) } \\
\hline$<15$ & $22(0,7)$ \\
\hline $15-19$ & $550(17,1)$ \\
\hline $20-34$ & $2.372(73,8)$ \\
\hline$\geq 35$ & $271(8,4)$ \\
\hline \multicolumn{2}{|l|}{ Escolaridade da mãe } \\
\hline Até primeiro grau completo & $731(23,4)$ \\
\hline $\begin{array}{l}\text { Segundo grau incompleto a } \\
\text { superior completo }\end{array}$ & $2.472(76,6)$ \\
\hline \multicolumn{2}{|l|}{ Cor da pele ${ }^{*}$} \\
\hline Branca & $567(17,9)$ \\
\hline Preta/negra & $426(13,2)$ \\
\hline Pardo & $2.179(67,7)$ \\
\hline Amarelo/oriental/indígena & $39(1,2)$ \\
\hline \multicolumn{2}{|l|}{ Situação conjugal } \\
\hline Com companheiro & $2.598(80,6)$ \\
\hline Sem companheiro & $617(19,4)$ \\
\hline \multicolumn{2}{|l|}{ No de filhos ${ }^{\star}$} \\
\hline$\leq 2$ & $2.597(80,8)$ \\
\hline$>2$ & $607(19,2)$ \\
\hline \multicolumn{2}{|l|}{ Atividade remunerada } \\
\hline Sim & $1.101(34,4)$ \\
\hline Não & $2.114(65,6)$ \\
\hline \multicolumn{2}{|l|}{ Renda familiar $(\mathrm{SM})^{\star}$} \\
\hline Até 3 & $1.790(66,6)$ \\
\hline$\geq 3 \mathrm{e}<5$ & $439(16,0)$ \\
\hline$\geq 5$ & $440(17,4)$ \\
\hline \multicolumn{2}{|l|}{$\begin{array}{l}\text { Consumo de bebida alcoólica } \\
\text { durante a gravidez }\end{array}$} \\
\hline Sim & $449(14,0)$ \\
\hline Não & $2.766(86,0)$ \\
\hline \multicolumn{2}{|l|}{ Fumo durante a gravidez } \\
\hline $\begin{array}{l}\text { Fumante/parou durante } \\
\text { gravidez }\end{array}$ & $110(3,6)$ \\
\hline $\begin{array}{l}\text { Fumante/não parou durante } \\
\text { gravidez }\end{array}$ & $214(6,7)$ \\
\hline Não fumante & $2.891(89,7)$ \\
\hline
\end{tabular}

ficante e mantida no modelo foi ter até primeiro grau completo $(\mathrm{RR}=1,64 ; \mathrm{IC}=1,28-2,09)$. No segundo bloco, foram as variáveis não ter planejado a gravidez $(\mathrm{RR}=1,42$; $\mathrm{IC}=1,13-1,79) \mathrm{e}$ consumo de bebida alcoólica durante este período $(\mathrm{RR}=1,42$; $\mathrm{IC}=1,13-1,79)$. Nenhuma variável do terceiro bloco permaneceu no modelo e no quarto bloco, o mais proximal, foram signi-
Tabela 3. Características dos recém-nascidos e da vida reprodutiva das mulheres estudadas, São Luís, 2013.

\begin{tabular}{|c|c|}
\hline Variáveis & $\mathbf{N}(\%)$ \\
\hline \multicolumn{2}{|l|}{ Idade da primeira gravidez } \\
\hline$<15$ & $125(4,0)$ \\
\hline $15-19$ & $1.387(43,1)$ \\
\hline$\geq 20$ & $1.703(52,9)$ \\
\hline \multicolumn{2}{|l|}{ Gravidez atual planejada* } \\
\hline Sim & $1.016(31,7)$ \\
\hline Não & $2.195(68,3)$ \\
\hline \multicolumn{2}{|c|}{$\begin{array}{l}\text { Ameaça de aborto na gravidez } \\
\text { atual }^{*}\end{array}$} \\
\hline Sim & $239(7,5)$ \\
\hline Não & $2.934(92,5)$ \\
\hline \multicolumn{2}{|l|}{ Tipo de parto } \\
\hline Normal & $1.640(50,7)$ \\
\hline Cesárea & $1.575(49,3)$ \\
\hline \multicolumn{2}{|c|}{ Tipo de financiamento do parto } \\
\hline Não SUS & $459(14,9)$ \\
\hline SUS & $2.756(85,1)$ \\
\hline \multicolumn{2}{|c|}{ Idade gestacional do recém-nascido } \\
\hline$<37$ & $225(14,1)$ \\
\hline$\geq 37$ & $1373(85,9)$ \\
\hline \multicolumn{2}{|l|}{ Apgar $5^{*}$} \\
\hline$<7$ & $36(1,2)$ \\
\hline$\geq 7$ & $3.137(98,8)$ \\
\hline \multicolumn{2}{|c|}{$\begin{array}{l}\text { Outra gravidez após o nascimento } \\
\text { da criança avaliada }\end{array}$} \\
\hline Sim & $282(8,9)$ \\
\hline Não & $2.933(91,1)$ \\
\hline
\end{tabular}

ficantes as variáveis sintomas de estresse $(\mathrm{RR}=$ $1,88$; IC $=1,51-2,33)$ e de depressão na mãe ( $R R$ $=2,00 ; \mathrm{IC}=1,57-2,56$ ).

Ao final da análise, verificou-se que a baixa escolaridade da mãe, sintomas de estresse e de depressão maternos foram fortemente associados à ocorrência de prejuízos na relação mãe-filho $(\mathrm{p} \leq 0,001)$. Não ter planejado a gravidez $(\mathrm{p}=$ $0,003)$ e o consumo de bebida alcoólica durante este período $(\mathrm{p}=0,015)$ também foram associados como fatores de risco.

\section{Discussão}

Neste estudo, a prevalência de prejuízos na relação mãe-filho foi de $12,6 \%$ e esteve associada à menor escolaridade, não ter planejado a gravidez, ter consumido bebida alcóolica durante esta, 
Tabela 4. Análise hierarquizada dos fatores associados a prejuízos na relação mãe-filho, São Luís, 2013.

\begin{tabular}{|c|c|c|c|c|c|c|c|c|}
\hline \multirow[b]{2}{*}{ Variáveis } & \multicolumn{2}{|c|}{ Nível Distal } & \multicolumn{2}{|c|}{ Nível Intermediário I } & \multicolumn{2}{|c|}{ Nível Intermediário II } & \multicolumn{2}{|c|}{ Nível Proximal } \\
\hline & RR (IC95\%) & $\begin{array}{c}\text { p- } \\
\text { valor }\end{array}$ & RR (IC95\%) & $\begin{array}{c}\text { p- } \\
\text { valor }\end{array}$ & RR (IC95\%) & $\begin{array}{c}\text { p- } \\
\text { valor }\end{array}$ & RR (IC95\%) & $\begin{array}{c}\text { p- } \\
\text { valor }\end{array}$ \\
\hline \multicolumn{9}{|l|}{ Escolaridade materna } \\
\hline $\begin{array}{l}\text { Até primeiro grau } \\
\text { completo }\end{array}$ & $1,64(1,28-2,09)$ & $<0,001$ & $1,51(1,23-1,86)$ & $<0,001$ & $1,41(1,05-1,89)$ & 0,021 & $1,44(1,19-1,74)$ & $<0,001$ \\
\hline $\begin{array}{l}\text { Segundo grau } \\
\text { incompleto a } \\
\text { superior completo }\end{array}$ & 1 & & 1 & & 1 & & 1 & \\
\hline \multicolumn{9}{|l|}{$\begin{array}{l}\text { Planejamento da } \\
\text { gravidez }\end{array}$} \\
\hline Sim & & & 1 & & 1 & & 1 & \\
\hline Não & & & $1,42(1,13-1,79)$ & 0,003 & $1,55(1,13-2,13)$ & 0,007 & $1,40(1,12-1,74)$ & 0,003 \\
\hline \multicolumn{9}{|l|}{$\begin{array}{l}\text { Consumo de bebida } \\
\text { alcoólica }\end{array}$} \\
\hline Sim & & & $1,42(1,13-1,79)$ & 0,003 & $1,41(1,02-1,93)$ & 0,035 & $1,25(1,00-1,56)$ & 0,015 \\
\hline Não & & & 1 & & 1 & & 1 & \\
\hline \multicolumn{9}{|l|}{ Sintomas de estresse } \\
\hline Sim & & & & & & & $1,88(1,51-2,33)$ & $<0,001$ \\
\hline Não & & & & & & & 1 & \\
\hline \multicolumn{9}{|l|}{ Sintomas de depressão } \\
\hline Sim & & & & & & & $2,00(1,57-2,56)$ & $<0,001$ \\
\hline Não & & & & & & & 1 & \\
\hline
\end{tabular}

IC - Intervalo de Confiança, RR - Risco relativo.

sintomas de estresse e de depressão maternos como fatores de risco.

Os achados relativos a prejuízos na relação mãe-filho foram mais prevalentes que em outros estudos realizados no Japão, Alemanha e Bélgica, com valores de $7,7 \%, 7,1 \%$ e $8,5 \%$, respectivamente. Esses estudos foram realizados com amostras da população geral e utilizaram o $P B Q$, sendo um deles, Van Bussel et al..$^{33}$, longitudinal. Porém, foram desenvolvidos em países com elevados indicadores sociais e abordaram as mães em fases mais precoces, variando entre duas semanas e três meses após o parto ${ }^{21,32,33}$.

Outro estudo, desenvolvido por Edhborg et al. ${ }^{15}$ em Bangladesh, encontrou prevalência de $11 \%$ quanto a prejuízos na relação mãe-filho utilizando o $P B Q$ em três momentos diferentes - terceiro trimestre da gestação, no dia do parto e 2 a 3 meses após este. Destaca-se que um terço dessas mães apresentava sintomas depressivos. Apesar da diferença metodológica no período de avaliação da relação, estes achados corroboram o presente estudo, sugerindo que condições de vida menos favoráveis podem se traduzir em maiores prejuízos na relação mãe-filho.
Reforçando esta ideia, verificou-se que menor escolaridade materna, variável do nível mais distal de determinação, esteve fortemente associada como risco à ocorrência de prejuízos na relação mãe-filho, permanecendo associada mesmo após ajuste pelas variáveis dos demais níveis.

Ferreira e Lima ${ }^{8}$ e Popp et al. ${ }^{9}$ destacam que mulheres inseridas nestes contextos apresentam maior incidência de padrões negativos quanto à capacidade de responder às necessidades da criança. Há maior dificuldade em reconhecer e responder aos sinais da criança, maior negatividade verbal, baixo calor afetivo, menor envolvimento e menos suporte às atividades da criança. Os autores ressaltam, ainda, que tais condições poderiam influenciar negativamente a qualidade da interação materna por constituírem fonte de estresse familiar ${ }^{8,9}$.

Figueiredo et al. ${ }^{34}$ retrataram que mulheres com nove ou mais anos de estudo apresentam mais emoções positivas em relação ao filho e estabelecem melhor relação com este. A educação dos pais pode oferecer benefícios para além dos recursos materiais viabilizados pela renda, pois estes podem ter maior acesso a informações que 
podem influenciar o cuidado parental e as necessidades de desenvolvimento das crianças ${ }^{35}$.

Klaus et al. ${ }^{19}$ referem que o planejamento da gravidez também interfere no estabelecimento da relação mãe-filho. Neste estudo, a ausência de planejamento da gravidez foi referida por $31,7 \%$ das mulheres e representou risco a prejuízos na relação mãe-filho em todos os níveis de análise.

A maternidade traz várias mudanças à vida da mulher e quando ocorre em momento não adequado, torna-se um evento estressante. $\mathrm{O}$ desejo e a intenção de engravidar são elementos que compõem o planejamento de uma gravidez ${ }^{36}$. Segundo Brockington et al. ${ }^{30}$ a gravidez não desejada combinada com a falta de interação com o feto estão fortemente associadas à rejeição do bebê.

Entretanto, a reação inicial de rejeição ao bebê no caso da gravidez não planejada pode não perdurar e dar lugar a uma atitude predominante de aceitação e vice-versa ${ }^{37}$.

Pirotta e Schor $^{38}$ referem que as mulheres levam em consideração seus contextos de vida ao escolher o melhor momento para sua gravidez, avaliando se as circunstâncias pessoais e/ou profissionais estão propícias para a chegada de uma criança.

O consumo de bebida alcóolica durante a gravidez foi referido por $14,0 \%$ das mulheres e esteve associado a prejuízos na relação mãe-filho. Não foram encontrados outros estudos que abordem esta associação. Alguns trabalhos avaliam o consumo de bebida alcóolica entre gestantes com estresse, sintomas depressivos e ansiosos ${ }^{39,40}$, porém não abordam a associação desse hábito com a relação mãe-filho.

O terceiro nível incluiu variáveis biológicas (Apgar no quinto minuto e idade gestacional) e uma relacional (outra gravidez após o nascimento da criança avaliada), que não estiveram associadas a prejuízos na relação mãe-filho. Fatores como esses são potencialmente influentes na relação mãe-filho ${ }^{41}$. Era esperado que o nascimento pré-termo estivesse associado com prejuízos nesta relação, considerando que a necessidade de hospitalização de boa parte destes bebês logo após o nascimento resulta na separação precoce da mãe e na privação dos cuidados maternos iniciais repercutindo na dificuldade da aproximação e da formação de vínculo entre ambos ${ }^{41}$. Porém, o ponto de corte em 37 semanas pode ter comprometido tal associação.

A falta de associação entre Apgar no quinto minuto e prejuízos na relação mãe-filho pode ter ocorrido devido ao pequeno número de crianças com Apgar muito baixo, influenciando a análise estatística. Não foi observada também, neste estudo, associação entre outra gravidez após o nascimento da criança avaliada e prejuízos na relação mãe-filho, apesar de ser retratado, entre os poucos estudos existentes sobre esta temática, uma diminuição acentuada nas interações mãe -primogênito, na atenção materna e no tempo que ocupavam em momentos conjuntos ${ }^{42-44}$.

Uma proporção substancial de mulheres que experimentam o estresse na gravidez ou durante o período do pós-parto continua a ter sintomas ao longo dos primeiros anos de seu filho ${ }^{45}$. Além disso, estudos têm demonstrado que estas mães podem ter dificuldades para formação de víncu$1 \mathrm{l}^{46}$, corroborando os achados deste estudo em que mulheres com sintomas de estresse tiveram risco de 1,88 vezes maior para prejuízos na relação mãe-filho durante o segundo e terceiro anos de vida da criança em comparação àquelas que não o apresentavam.

Benzies et al. ${ }^{46}$ e Brand e Brennan ${ }^{47}$ referem que a exposição da mãe a diversas situações estressoras pode gerar prejuízos à relação mãe-filho. Vale destacar que a maternidade se constitui em uma nova etapa do ciclo de vida familiar, que tende a gerar estresse devido a uma infinidade de mudanças bruscas ${ }^{48}$. Nesse contexto, a mulher tende a se sentir mais insegura tanto pelas mudanças ocorridas como pelo fato dela deixar de ser o centro de sua própria vida ${ }^{49}$.

Os achados de Benzies et al. ${ }^{16}$ sugerem que filhos de mães com estresse devem receber acompanhamento adicional para reduzir o risco de atraso no desenvolvimento global, dado que o vínculo inconsistente pode também exercer influência direta em comprometimentos emocionais e comportamentais nas crianças ${ }^{16,46}$.

Outro aspecto que potencialmente afeta negativamente a relação mãe-filho, também ligado a problemas de saúde mental materna, é a depressão $0^{15,19,30,50}$. Brockington et al. ${ }^{30}$ avaliaram distúrbios graves na relação mãe-bebê entre mulheres com depressão pós-parto, na Inglaterra, utilizando a Entrevista Birmingham para a Saúde Mental Materna. Estes identificaram que 29\% das mães entrevistadas no serviço de Birmingham e $22 \%$ no de Christchurch apresentaram ameaça de rejeição ou rejeição estabelecida em relação aos bebês.

Neste estudo, apresentar sintomas de depressão esteve fortemente associado com prejuízos na relação mãe-filho, representando risco 2,00 vezes maior. Este achado corrobora o de outros autores que investigaram a relação por meio do $P B Q$ em 
que sintomas depressivos constituíram preditores mais fortes de prejuízos nesta relação ${ }^{22,50}$.

Mães depressivas geralmente apresentam tristeza, necessidade de isolamento, baixa autoestima e elevados níveis de estresse ${ }^{51}$, que podem dificultar o investimento emocional no seu filho e a garantia dos cuidados que este necessita, repercutindo, desta forma, na manutenção do vínculo afetivo saudável ${ }^{30}$.

Entretanto, mães com depressão que são mais sensíveis podem apresentar comportamentos de maternagem e seus bebês serem menos propensos a efeitos negativos a longo prazo ${ }^{52}$.

Constituiu limitação deste estudo a acentuada perda de seguimento que é comum em estudos de coorte e que foi minimizada pela utilização da ponderação para análise dos dados. Ainda assim o tamanho da amostra foi suficiente para demonstrar as associações estudadas. As prevalências encontradas podem sofrer influência da reprodução social nas respostas ao $P B Q$ em que a mãe tenta transmitir uma imagem de si que seja positivamente valorizada pela sociedade ${ }^{21}$. Este fato pode mascarar o cenário real de comprometimento da relação mãe-filho.

O fato do $P B Q$ não ter sido validado para a população brasileira também poderia constituir uma desvantagem, porém apresentou-se com boa consistência interna verificada por meio do $\alpha$ de Cronbach. Além disso, este é o instrumento mais utilizado para diagnosticar problemas na relação mãe-filho ${ }^{28}$.

Por fim, constituíram pontos fortes desta pesquisa ser um estudo de coorte, realizado em amostra populacional, com a utilização de modelo hierarquizado que permitiu a investigação de associações entre variáveis de diversos níveis de influência para prejuízos na relação mãe-filho.

\section{Conclusão}

Na população estudada, a prevalência de prejuízos na relação mãe-filho foi elevada quando comparada a de países com altos indicadores sociais e esteve associada a elementos da saúde mental e à escolaridade, que constituíram risco para esta relação.

Estes resultados apontam para a importância da atenção voltada para saúde mental das mulheres ao longo da assistência pré-natal, de modo a prevenir a instalação ou o agravamento de problemas psicológicos que poderão repercutir negativamente na relação mãe-filho.

\section{Colaboradores}

MCV Cavalcante e AKTC França participaram da análise e interpretação dos dados para o trabalho, redação do artigo e aprovação da versão a ser publicada. F Lamy Filho e ZC Lamy participaram da concepção, delineamento e interpretação dos dados para o trabalho, revisão crítica do conteúdo intelectual e aprovação da versão a ser publicada. 


\section{Referências}

1. Brockington I. Maternal rejection of the young child: Present status of the clinical syndrome. Psychopathol 2011; 44(5):329-336.

2. Bowlby J. Apego - A natureza do vínculo: Apego e perda. São Paulo: Martins Fontes; 2009. (Volume. 1).

3. Brockington IF, Oates J, George S, Turner D, Vostanis P, Sullivan M, Loh C, Murdoch C. A screening questionnaire for mother-infant bonding disorders. Arch Women's Ment Health 2001; 3(4):133-140.

4. Stack DM, Serbin LA, Girouard N, Enns LN, Bentley VMN, Ledingham JE, Schwartzman AE. The quality of the mother-child relationship in high-risk dyads: Application of the Emotional Availability Scales in an intergenerational, longitudinal study. Dev Psychopathol 2012; 24(1):93-105.

5. Walker SP, Wochs TD, Gardner JM, Wasserman GA, Pollitt E, Carter JA, International Child Development Steering Group. Child development: Risk factors for adverse outcomes in developing countries. Lancet 2007; 369(9556):145-157.

6. Figueiredo B. Vinculação materna: Contributo para a compreensão das dimensões envolvidas no processo inicial de vinculação da mãe ao bebé. Rev Int Psicol Clin Salud 2003; 3(3):521-539.

7. Stack DM, Serbin LA, Enns LN, Ruttle P, Barrieau L. Parental effects on children's emotional development over time and across generations. Infant Young Child 2010; 23(1):52-69.

8. Ferreira T, Lima IA. Responsividade materna e risco psicossocial: Implicações práticas. Rev Amazônica 2012; VIII(1):33-52.

9. Popp T, Spinrad T, Smith C. The relation of cumulative demographic risk to mothers' responsivity and control: Examining the role of toodler temperament. Infancy 2008; 13(5):496-518.

10. Di Matteo MR, Morton SC, Lepper HS, Damush TM, Carney MF, Pearson M, Kahn KL. Cesarean childbirth and psychosocial outcomes: A meta- analysis. Health Psychol 1996; 15(4):303-324.

11. Moreira MEL, Gama SGN, Pereira APE, Silva AAM, Lansky S, Pinheiro RS, Gonçalves AC, Leal MC. Práticas de atenção hospitalar ao recém-nascido saudável no Brasil. Cad Saude Publica 2014; 30(Supl. 1):S128-S139.

12. Taylor A, Littlewood J, Adams D, Doré C, Glover V. Serum cortisol levels are related to moods of elation and dysphoria in new mothers. Psychiatry Res 1994; 54(3):241-247.

13. Taylor A, Fisk NM, Glover V. Mode of delivery and subsequent stress response. Lancet 2000; 355:120.

14. Brockington IF, Aucamp HM, Fraser C. Severe disorders of the mother-infant relationship: definitions and frequency. Arch Women's Ment Health 2006; 9(5):243251.
15. Edhborg M, Nasreen HE, Kabir ZN. Impact of postpartum depressive and anxiety symptoms on mothers' emotional tie to their infants 23 months postpartum: A population based study in rural Bangladesh. Arch Women's Ment Health 2011; 14(4):307-316.

16. Benzies K, Mychasiuk R, Tough S. What patterns of postpartum psychological distress are associated with maternal concerns about their children's emotional and behavioural problems at the age of three years? Early Child Dev Care. [periódico na Internet]. 2014 [acessado 2014 nov 16]. Disponível em: http://dx.doi. org/10.1080/03004430.2014.899592

17. Oliveira ASF. O Impacto dos estados emocionais negativos maternos na vinculação materna pós-natal em mães de bebés pré-termo [dissertação]. Lisboa: Universidade de Lisboa; 2012.

18. Brasil. Nascer no Brasil: Inquérito Nacional sobre Parto e Nascimento. A mãe sabe parir, e o bebê sabe como e quando nascer. Rio de Janeiro: Editora Fiocruz; 2014.

19. Klaus MH, Kennell JH, Klaus PH. Vínculo - Construindo as bases para um apego seguro e para a independência. Porto Alegre: Artes Médicas; 2000.

20. Rosenkrantz S, Aronson S, Huston A. Mother-infant relationship in single, cohabiting, and married families: a case for marriage? J Fam Psychol 2004; 18(1):5-18.

21. Reck C, Klier CM, Pabst K, Stehle E, Steffenelli U, Struben $\mathrm{K}$, Backenstrass $\mathrm{M}$. The German version of the Postpartum Bonding Instrument: Psychometric properties and association with postpartum depression. Arch Women's Ment Health 2006; 9(5):265-271.

22. Sockol LE, Battle CL, Howard M, Davis T. Correlates of impaired mother-infant bonding in a partial hospital program for perinatal women. Arch Women's Ment Health 2014; 17(5):465-469.

23. Cox JL, Holden JM, Sagovsky R. Detection of postnatal depression: Development of the 10-item Edinburgh Postnatal Depression Scale. Br J Psychiatry 1987; 150:782-786.

24. Santos IS, Matijasevich A, Tavares BF, Barros AJD, Botelho IP, Lapolli C, Magalhães PV, Barbosa AP, Barros FC. Validation of the Edinburgh Postnatal Depression Scale (EPDS) in a sample of mothers from the 2004 Pelotas Birth Cohort Study. Cad Saude Publica 2007; 23(11):2577-2588.

25. Ruschi GEC, Sun SY, Mattar R, Chambô Filho A, Zandonade E, Lima VJ. Aspectos epidemiológicos da depressão pós-parto em amostra brasileira. Rev Psiquiatr Rio Gd Sul 2007; 29(3):274-280.

26. Cohen S, Williamsom GM. Perceived Stress in a Probability Sample of United States. In: Spacapan S, Oskamp S. editors. The Social Psychology of Health: Claremont Symposium on applied social psychology. Newbury Park: Sage; 1988. p. 31-67. 
27. Luft CDB, Sanches SO, Mazo GZ, Andrade A. Versão brasileira da Escala de Estresse Percebido: tradução e validação para idosos. Rev Saude Publica 2007; 41(4):606-615.

28. Perrelli JGA, Zambaldi CF, Cantilino A, Sougey EB. Instrumentos de avaliação do vínculo entre mãe e bebê. Rev Paul Pediatr 2014; 32(3):257-265.

29. Nazaré B, Fonseca A, Canavarro MC. Postpartum Bonding Questionnaire: Estudo da versão portuguesa numa amostra comunitária. In: Ferreira AS, Verhaeghe A, Silva DR, Almeida LS, Lima R, Fraga S, editores. Actas do VIII Congresso Iberoamericano de Avaliação Psicológica/XV Conferência Internacional de Avaliação Psicológica: Formas e Contextos. Lisboa, Portugal: Sociedade Portuguesa de Psicologia; 2011. p. 1961-1973.

30. Brockington IF, Fraser C, Wilson D. The Postpartum Bonding Questionnaire: A validation. Arch Women's Ment Health 2006; 9(5):233-242.

31. Moehler E, Wiebel R, Brunner A, Reck C, Resch F. Maternal depressive symptoms in the postnatal period are associated with long-term impairment of mother-child bonding. Arch Women's Ment Health 2006; 9(5):273278.

32. Kaneko H, Honjo S. The Psychometric Properties and Factor Structure of the Postpartum Bonding Questionnaire in Japanese Mothers. Psychol 2014; 5(9):11351142.

33. Van Bussel JCH, Spitz B, Demyttenaere K. Three self-report questionnaires of the early mother-to-infant bond: reliability and validity of the Dutch version of the MPAS, PBQ and MIBS. Arch Women's Ment Health 2010; 13(5):373-384.

34. Figueiredo B, Costa R, Pacheco A, Pais A. Mother-to-infant emotional involvement at birth. Matern Child Health J 2009; 13(4):539-549.

35. Hoff E, Laursen B, Tardif T. Socioeconomic status and parenting. In: Borstien MH, editor. Handbook of Parenting. Mahweh: Lawrence Erlbaum Associated; 2002.

36. Morin P, Payette H, Moos MK, St-Cyr-Tribble D, Niyonsenga T, De Wals P. Measuring the intensity of pregnancy planning effort. Paediatr Perinat Epidemiol 2003; 17(1):97-105.

37. Maldonado MTP. Psicologia da gravidez, parto e puerpério. Vozes: Petrópolis; 1988.

38. Pirotta KMC, Schor N. Intenções reprodutivas e práticas de regulação da fecundidade entre universitários. Rev Saude Publica 2004; 38(4):495-502.

39. Spietz A, Kelly J. The importance of maternal mental health during pregnancy: theory, practice, and intervention. Public Health Nurs 2002; 19(3):153-155.

40. Pinheiro SN, Laprega MR, Furtado EF. Morbidade psiquiátrica e uso de álcool em gestantes usuárias do Sistema Único de Saúde. Rev Saude Publica 2005; 39(4):593-598.
41. Chiodi LC, Aredes ND, Scochi CGS, Fonseca LMM Educação em saúde e a família do bebê prematuro: uma revisão integrativa. Acta Paulista de Enfermagem 2012; 25(6):969-974.

42. Dunn J, Kendrick C. The arrival of a sibling: changes in patterns of interaction between mother and first-born child. J Child Psychol Psychiatry 1980; 21(2):119-132.

43. Field T, Reite M. Children's responses to separation from mother during the birth of another child. Child Development 1984; 55(4):1308-1316.

44. The firstborn's adjustment to the birth of a sibling: a longitudinal assessment. Child Development 1987; 58(2):341-355.

45. Horwitz SM, Briggs-Gowan MJ, Storfer-Isser A, Carter AS. Persistence of maternal depressive symptoms throughout the early years of childhood. J Women's Health 2009; 18(5):637-645.

46. Benzies K, Keown LA, Magill-Evans J. Immediate and sustained effects of par- enting on physical aggression in Canadian children to 6 years of age. Can J Psychiatry 2009; 54(1):73-82.

47. Brand SR, Brennan PA. Impact of antenatal and postpartum maternal mental illness: How are the children? Clin Obstet Gynec 2009; 52(3):441-455.

48. Terry DJ, Mayocchi L, Hynes GJ. Depressive symptomology in new mothers: a stress and coping perspective. J Abnorm Psychol 1996; 105(2):220-231.

49. Falceto OG. A influência de fatores psicossociais na interrupção precoce do aleitamento materno [tese]. Porto Alegre: Universidade Federal do Rio Grande do Sul; 2002.

50. Paris R, Bolton RE, Weinberg MK. Postpartum depression, suicidality, and mother-infant interactions. Arch Women's Mental Health 2009; 12(5):309-332.

51. Francisco VL, Pires A, Pingo S, Henriques R, Esteves MA, Valada MJ. A depressão materna e o seu impacto no comportamento parental. Aná Psicológica 2007; 25(2):229-239.

52. Boyd D, Bee H. A criança em crescimento. Porto Alegre: Artes Médicas; 2011.

Artigo apresentado em 21/05/2015

Aprovado em 22/11/2015

Versão final apresentada em 24/11/2015 
